\title{
HIPOTIROIDISMO Y RIESGO DE ABORTO
}

\section{Hypothyroidism and risk of miscarniage}

\author{
Carlos Arturo Vivas, M.D. *, Jenny Soraya Cárdenas**, Sandra Milena Cardozo**, \\ Katherine Carvajal-Canizales**, Juan Camilo Cifuentes**
}

Recibido: noviembre 10/08 - Aceptado: mayo 19/09

\section{RESUMEN}

Objetivo: revisar, en la literatura disponible, aquellas evidencias que muestren la asociación entre hipotiroidismo y aborto.

Metodología: se realizó una búsqueda bibliográfica utilizando la base de datos especializada Medline y los buscadores Pubmed y Ovid para identificar literatura relevante.

Resultados: aunque varios estudios mostraron un aumento en el riesgo de aborto en las pacientes con hipotiroidismo clínico, hipotiroidismo subclínico y en pacientes eutiroideas con anticuerpos antitiroideos, fue en este último grupo de mujeres en donde se evidenció una fuerte asociación entre el hipotiroidismo y el aborto. Por lo tanto, se recomienda realizar el diagnóstico y tratamiento temprano del hipotiroidismo clínico y subclínico en las mujeres embarazadas, así como la búsqueda de anticuerpos antitiroideos en el grupo de abortadoras recurrentes; y aunque algunos trabajos han demostrado una disminución en el riesgo de aborto utilizando suplencia con levotiroxina en las pacientes con anticuerpos antitiroideos, aún no se puede recomendar dicha terapia en este grupo de pacientes.

Conclusión: se requieren estudios adicionales con un mayor número de pacientes y un adecuado diseño para definir claramente la asociación entre

* Especialista en Ginecología y Obstetricia. Profesor de la Universidad del Tolima, Facultad de Ciencias de la Salud, Programa de Medicina. Ibagué (Colombia).Correo electrónico: cavira694@hotmail.com o unifertil@ yahoo.com

** Estudiante de quinto año de Medicina. Universidad del Tolima. Ibagué (Colombia) aborto y enfermedad tiroidea, y así poder establecer terapias adecuadas.

Palabras clave: embarazo, hipotiroidismo, aborto espontáneo, autoinmunidad, autoanticuerpos, tiroiditis, tiroxina.

\section{SUMMARY}

Objective: this article was aimed at searching the literature for an association between hypothyroidism and miscarriage.

Methodology: a search was made on the Medline electronic database, using Pubmed and Ovid for identifying relevant material.

Results: some studies showed an increased risk of miscarriage in patients suffering from overt hypothyroidism and subclinical hypothyroidism, and in euthyroid patients having antithyroid antibodies; a strong association was seen in the latter group. Early diagnosis and treatment of pregnant women having subclinical, overt hypothyroidism and the search for antithyroid antibodies is highly recommended in recurrent miscarriage. Despite some studies having shown a decrease in the risk of miscarriage when levothyroxine treatment was used with patients having antithyroid antibodies, it cannot be recommended yet.

Conclusion: more studies using an increased number of patients have to be carried out to define a true association between miscarriage and thyroid disorders and thus establish suitable treatment.

Key words: pregnancy, hypothyroidism, subclinical hypothyroidism, miscarriage, autoimmunity, thyroid auto antibodies, thyroiditis, thyroxine. 


\section{INTRODUCCIÓN}

El hipotiroidismo se puede definir como cualquier alteración estructural o funcional que interfiere en la producción, secreción o acción periférica de las hormonas tiroideas. El hipotiroidismo subclínico, por su parte, es conocido como un incremento en los niveles de la hormona secretora de tirotropina (TSH, por sus siglas en inglés) asociado con niveles de hormonas $\mathrm{T}_{4}$ y $\mathrm{T}_{3}$ normales. De otro lado, los valores de TSH $>10 \mathrm{mU} / \mathrm{L}$ en el hipotiroidismo manifiesto se encuentran asociados a cantidades bajas de tiroxina y a la presencia de síntomas. ${ }^{1}$ La mayoría de los casos de hipotiroidismo corresponden a hipotiroidismo primario y su causa más frecuente, en regiones donde no existe déficit de yodo, es la tiroiditis autoinmune crónica o de Hashimoto, con una incidencia de 15\% - 60\% de todos los casos de hipotiroidismo. ${ }^{2}$

En la tiroiditis autoinmune, la respuesta antitiroidea comienza con la activación de las células $T$ ayudadoras antígeno-específicas, las cuales inducen la secreción de anticuerpos antitiroideos por parte de los linfocitos B. ${ }^{3}$ La antitiroglobulina (anti-Tg) y la antiperoxidasa (TPO) son los anticuerpos que se miden con mayor frecuencia y su presencia se asocia con daño tiroideo e infiltración linfocítica de la tiroides. Los TPO, por ejemplo, fijan complemento y tienen un efecto citotóxico sobre las células tiroideas. ${ }^{3}$ Asimismo, se han observado anticuerpos antireceptores de TSH que podrían contribuir a la disfunción tiroidea. ${ }^{4,5}$

Por otro lado, actualmente se considera que la tasa de pérdidas gestacionales después de la implantación es de 31\%, ${ }^{6}$ y la de los embarazos reconocidos clínicamente, de 15\% - 20\%. ${ }^{6,7}$ Además, las modificaciones en la foliculogénesis y la ovulación pueden reflejarse en anomalías durante el desarrollo embrionario, alteraciones en la receptividad uterina y en el posterior funcionamiento del cuerpo lúteo. Del mismo modo, múltiples trastornos endocrinos se han asociado con un aumento en el riesgo de aborto, tales como: hipersecreción de la hormona luteinizante (LH, por sus siglas en inglés), hiperandrogenismo, síndrome de ovario poliquístico, hiperprolactinemia y el hipotiroidismo. ${ }^{8,9}$

Sin embargo, aunque la asociación entre hipotiroidismo y aborto ha sido mencionada en la literatura disponible sobre estos temas, ésta relación no está claramente demostrada. Nuestro propósito, por lo tanto, es buscar evidencia que soporte éste fenómeno, y de ser así, poder conocer las variables involucradas. De este modo, se podrá justificar la búsqueda del hipotiroidismo subclínico y resaltar la importancia del tratamiento temprano de esta enfermedad en la mujer embarazada.

\section{METODOLOGÍA}

Se realizó una búsqueda bibliográfica utilizando la base de datos especializada Medline y los buscadores Pubmed y Ovid para identificar literatura relevante. Los términos de búsqueda empleados fueron: hypothyroidism, subclinic hypothyroidism, miscarriage, autoimmunity, thyroid autoantibodies.

\section{RESULTADOS DE LA BÚSQUEDA Metabolismo de la tiroides y embarazo}

Los cambios hormonales y metabólicos que se producen durante el embarazo dan lugar a alteraciones bioquímicas de la función tiroidea, generando niveles hormonales que cambian de acuerdo con las diferentes etapas del embarazo. Sus efectos pueden ser transitorios o persistir hasta el final de la gestación. ${ }^{10}$

En el organismo, las reservas de yodo se encuentran representadas por su ingesta diaria y por el catabolismo de las hormonas tiroideas, lo cual hace que se produzca un equilibrio dinámico entre dos órganos principales, la tiroides y el riñón. Por otro lado, durante el embarazo se produce un aumento en la filtración glomerular y, por consiguiente, un aumento en la depuración de yodo, lo que conlleva a una disminución del yodo circulante que es compensada con el incremento de la funcionalidad de la glándula tiroidea y de la recaptación de yodo. ${ }^{11}$ Adicionalmente, en la placenta, por acción de la desyodasa tipo 3 se produce un aumento en el 
recambio de $\mathrm{T}_{4}$, convirtiendo $\mathrm{T}_{4}$ en $\mathrm{T}_{3}$ reversa, y $\mathrm{T}_{3}$ en 3,3 diyodotironina. ${ }^{10,12}$

El incremento de estrógenos, propio del embarazo, duplica la globulina fijadora de tiroxina (TGB, por sus siglas en inglés) debido a la estimulación de la síntesis hepática y a la disminución de su depuración por aumento de la glucosilación. ${ }^{11}$ En 1977, Glinoer demostró que las concentraciones elevadas de estrógenos en monos Rhesus estimulan los hepatocitos elevando la producción de TGB en el hígado. ${ }^{13}$ Estos niveles altos de TGB aumentan la unión de $\mathrm{T}_{4}$, lo que disminuye la concentración de $\mathrm{T}_{4}$ libre e incrementa la de $\mathrm{T}_{4}$ total; posteriormente, estos eventos se ven reflejados en un aumento de TSH (dentro de los valores normales) que produce un equilibrio en la función tiroidea materna. ${ }^{11}$

Otro hecho propio del embarazo es el estímulo del anticuerpo receptor de TSH por parte de la Gonadotropina Coriónica Humana (HCG, por sus siglas en inglés), la cual se eleva precozmente en la gestación y alcanza un pico a las 12 semanas. Ésto se debe a la similitud que presentan la HCG y la TSH en su subunidad $\beta$ y a la homología que muestran sus respectivos receptores. ${ }^{11}$ Como consecuencia, este efecto puede aumentar levemente la $\mathrm{T}_{4}$ y $\mathrm{T}_{3}$ libre e inducir la reducción parcial de la TSH, lo que se normaliza alrededor de la vigésima semana de embarazo. ${ }^{11,14}$ De otra parte, durante las etapas finales del embarazo, el yodo disponible de la madre es transferido al feto, lo que contribuye a la disminución de los niveles circulantes de éste en el suero materno. De este modo, en una mujer no embarazada, la ingesta de yodo en niveles normales es de $100-150 \mu \mathrm{g} /$ día, mientras que en las mujeres embarazadas se necesitan hasta $200 \mu \mathrm{g} /$ día. $^{12}$

Por otro lado, la degradación de las hormonas tiroideas se lleva a cabo por la acción de tres enzimas, la desyodasa tipos 1, 2, y 3. La desyodasa tipo 1 es la encargada de degradar la $\mathrm{T}_{4}$ en $\mathrm{T}_{3}$, por lo cual es la responsable del aumento sérico de $\mathrm{T}_{3}$ total. $\mathrm{La}$ desyodasa tipo 2, por su parte, tiene cierto grado de selectividad por $\mathrm{T}_{4}$ y $\mathrm{T}_{3}$ reversa y se expresa en algunos órganos y tejidos como la hipófisis, el cerebro, el tejido adiposo y la placenta. La desyodasa tipo 3 se encuentra en grandes cantidades en la placenta y toma como sustrato $\mathrm{T}_{4}$ para convertirlo en $\mathrm{T}_{3}$ reversa, lo que explica los valores disminuidos de $\mathrm{T}_{4}$, y aumentados de $\mathrm{T}_{3}$ reversa en la economía tiroidea fetal. ${ }^{10,11}$

En resumen, la disminución de las concentraciones séricas de tiroxina libre en la mujer embarazada puede deberse a: ${ }^{10}$

- Incremento sérico en las concentraciones de TGB.

- Degradación de la hormona tiroidea por la placenta.

- Transferencia de tiroxina de la madre al feto.

- Aumento de la depuración de tiroxina por la madre.

Además, las hormonas tiroideas $\mathrm{T}_{3} \mathrm{y}_{4}$ se elevan durante el primer trimestre del embarazo y los niveles de tirotropina disminuyen. ${ }^{10,14}$ Estos cambios ocurren hasta las semanas 10 y 12 de gestación como respuesta a la demanda y alcanzan su pico máximo hacia la semana 20, cuando sus valores se estabilizan y se mantienen hasta el final del embarazo. Dichas modificaciones son necesarias para lograr un equilibrio en la economía hormonal tiroidea en respuesta a las necesidades concepcionales, lo que implica un constante reajuste de la TGB y $\mathrm{T}_{4}$ durante las fases iniciales del embarazo.

\section{HIPOTIROIDISMO EN EL EMBARAZO}

En mujeres con función tiroidea normal, la producción de tiroxina puede aumentar para compensar el decremento de las concentraciones de tiroxina sérica libre. En el caso de las mujeres embarazadas eutiroideas, la TSH no sufre grandes modificaciones y sus valores se conservan dentro de límites normales; por el contrario, en mujeres con déficit de yodo o tiroiditis autoinmune, los niveles de TSH se elevan significativamente dejando al descubierto mecanismos de adaptación deficientes. ${ }^{11}$ 
El embarazo en mujeres con hipotiroidismo avanzado es poco frecuente debido a la asociación que éste presenta con anovulación y porque, aún si se logra la fecundación, es muy posible que la frecuencia de aborto aumente en el primer trimestre. ${ }^{14}$ Sin embargo, las mujeres con hipotiroidismo moderado a leve no tratado pueden quedar en embarazo, pero el riesgo fetal (aborto, mortinato o parto prematuro) y materno (hipertensión del embarazo) son mayores. No obstante, el verdadero impacto del hipotiroidismo sobre el riesgo de aborto es difícil de separar de la acción de los anticuerpos antitiroideos, ya que la sóla presencia de éstos en mujeres eutiroideas, incrementa también el riesgo de aborto. ${ }^{15}$

El feto carece de actividad tiroidea hasta la semana 12 de gestación, y durante todo ese tiempo depende totalmente de la hormona tiroidea materna. Hay dos consecuencias indeseables cuando se tienen valores de $\mathrm{T}_{4}$ bajos; la primera es el riesgo de complicaciones obstétricas, y la segunda es el riesgo de alteraciones en el desarrollo cerebral fetal. El inicio del desarrollo cerebral fetal depende de la disponibilidad de $\mathrm{T}_{4}$ en el tejido embrionario, por lo que prevenir la hipotiroxemia materna temprana previene los defectos en el neurodesarrollo que pueden llegar a ocasionar un bajo coeficiente intelectual y un alto riesgo de parálisis cerebral. ${ }^{16}$

Por consiguiente, el diagnóstico de hipotiroidismo durante el embarazo es crucial debido a los efectos adversos potenciales, tanto para la madre como para el niño; sólo 20\% - 30\% de las pacientes hipotiroideas desarrollan síntomas consistentes con la enfermedad, mientras que la mayoría que padece hipotiroidismo subclínico son asintomáticas.

Otras complicaciones obstétricas importantes son: hipertensión gestacional (que ocurre en mujeres hipotiroideas en 36\% de los casos, en pacientes con hipotiroidismo subclínico en 25\% de los casos y en la población general en $8 \%$ de los casos), ${ }^{17}$ abruptio de placenta, anemia y hemorragia posparto. ${ }^{1,18}$

\section{HIPOTIROIDISMO Y ABORTO ESPONTÁNEO}

El embarazo puede afectar el curso de la enfermedad tiroidea y la enfermedad tiroidea puede, a su vez, afectar el curso del embarazo. Las mujeres embarazadas con hipotiroidismo tienen un riesgo dos a cuatro veces mayor de aborto, en algunos casos asociados con la presencia de anticuerpos antitiroideos. ${ }^{11,19}$ En 2006, LeBeau y Mande ${ }^{20}$ llevaron a cabo un estudio para examinar las complicaciones en el embarazo en mujeres con niveles de TSH elevados. Los hallazgos indicaron que las mujeres que presentan hipotiroidismo durante el embarazo tienen 3,8\% de riesgo de aborto tardío en comparación con $0,9 \%$ de riesgo en mujeres con función tiroidea normal. Igualmente, en este estudio se encontró que 6 de cada 100 abortos tardíos pueden ser atribuidos a una deficiencia tiroidea durante el embarazo. Asimismo, en 209 de 9.403 pacientes embarazadas se encontraron niveles de TSH mayores a $6 \mathrm{mU} / \mathrm{L}$ (2,2\% de los casos). La tasa de muerte fetal tardía fue significativamente más alta en estos embarazos (8 de 209 ó 3,8\% de los casos) comparada con la encontrada en las mujeres con niveles de TSH < $6 \mathrm{mU} / \mathrm{L}$ (0,9\% de los casos). Además, las tasas de muerte fetal se incrementaban a medida que los niveles de TSH aumentaban. De este modo, de las 37 mujeres con niveles de TSH mayores a $10 \mathrm{mU} / \mathrm{L}$, las muertes fetales ocurrieron en $8,1 \%$ de los casos (20 mujeres).

Abalovich, en el año 2002, ${ }^{21}$ en un estudio retrospectivo, evaluó la tasa de abortos en mujeres con hipotiroidismo previo al embarazo, en las cuales se ajustó la dosis de hormona tiroidea. En un grupo de 27 pacientes, se administraron las dosis adecuadas de levotiroxina, llevándolas a un estado eutiroideo, mientras que en otro grupo de 24 mujeres la dosis de levotiroxina no fue adecuada con la subsecuente permanencia de su estado hipotiroideo. Como resultado, en el grupo de mujeres con tratamiento inadecuado, la tasa de abortos fue de $60 \%$ en las madres con hipotiroidismo clínico y de $71 \%$ en las madres con hipotiroidismo subclínico. Además, la 
prevalencia de parto pretérmino se vio incrementada. Por el contrario, en el grupo de mujeres con dosis adecuadas de levotiroxina, la tasa de abortos fue de $4 \%$; incluso las mujeres a las que se les administró tempranamente restitución de levotiroxina en forma adecuada, la tasa de abortos cayó a $0 \%$, permitiendo inferir que un tratamiento temprano con levotiroxina podría disminuir el riesgo de aborto. ${ }^{21}$

\section{AUTOINMUNIDAD TIROIDEA Y ABORTO}

Además de la identificación de factores genéticos, anatómicos o tóxicos como inductores de aborto espontáneo, a través del tiempo se ha presentado evidencia cada vez más sólida que soporta la asociación entre las enfermedades autoinmunes y el aborto recurrente. En una revisión realizada en 2003 de trece trabajos que estudiaron la relación entre la enfermedad tiroidea autoinmune y el aborto, Poppe sugiere que existe una fuerte asociación entre la presencia de anticuerpos antitiroideos y el riesgo de aborto. ${ }^{19}$

Similarmente, un metaanálisis realizado en el año 2004 que abarcó todos los estudios longitudinales, de casos y controles desde 1990 sobre la asociación entre aborto y enfermedad tiroidea autoinmune, confirma ampliamente que el riesgo relativo de aborto es tres veces mayor en mujeres con autoinmunidad tiroidea que en las mujeres sanas. ${ }^{22}$ En el caso de la madre eutiroidea, con presencia de anticuerpos antitiroideos, el aborto recurrente podría estar asociado a una menor habilidad de la función tiroidea para adaptarse adecuadamente a los cambios asociados con el embarazo debido a que las reservas funcionales de la tiroides podrían ser insuficientes para suplir la demanda que se presenta en el embarazo, lo cual puede reflejar el carácter crónico del padecimiento autoinmune así no se hayan presentado manifestaciones clínicas. ${ }^{19,23}$

De igual manera, Tartakover demostró, en ratones, la asociación entre la presencia de anticuerpos anti-Tg y la alteración fetal y placentaria. ${ }^{24}$ El hallazgo de la relación entre los autoanticuerpos tiroideos y el aborto no excluye la posibilidad de que éstos sean marcadores de un estado autoinmune aún no identificado que actúe contra la unidad fetoplacentaria. Esta idea se sustenta al observar que las mujeres con aborto recurrente tienen un número incrementado de linfocitos B CD5/20 comparadas con mujeres con un embarazo normal o con un sólo aborto, mientras que en otras mujeres con anticuerpos antitiroideos se encuentran linfocitos $T$ con anormalidades en su función; dichas mujeres tienen un número mayor de linfocitos $\mathrm{T}$ endometriales y estos linfocitos producen menos IL-4 e IL-10 pero más INF gamma. ${ }^{22}$ Un estudio realizado por Wilson en 1999, que comparó los niveles de anticuerpos antiperoxidasa, reveló que aquellas mujeres que tuvieron abortos recurrentes presentaban títulos más altos de anticuerpos que aquellas que llevaron su embarazo a término. ${ }^{23}$ Además, se han encontrado otros factores no inmunológicos, como la edad, que intervienen en la ocurrencia de aborto en las madres con anticuerpos antitiroideos positivos; se reconoce que mujeres mayores de 35 años tienen mayor riesgo de aborto. ${ }^{22}$ Es importante destacar que las mujeres con anticuerpos antitiroideos tienen la tendencia a quedar embarazadas a mayor edad (35 años o más), permitiendo la combinación de dos factores de riesgo independientes (edad y autoanticuerpos tiroideos) para aborto recurrente. ${ }^{19,22}$

Del mismo modo, Muller, ${ }^{25}$ en 1999, realizó un estudio en 173 mujeres que iban a ser sometidas a fertilización in vitro (FIV), realizándoles medición de niveles de anticuerpos antiperoxidasa antes de iniciar el tratamiento. Los resultados demostraron un mayor número de abortos en aquellas mujeres que eran anti-TPO positivas (33\% vs. 19\%). Igualmente, $\mathrm{Negro}^{26}$ realizó un estudio con 984 mujeres donde 115 fueron positivas para anti-TPO, las cuales se dividieron aleatoriamente en dos grupos: uno de ellos recibió tratamiento de suplencia con levotiroxina $(n=57)$ de acuerdo con los niveles de $\mathrm{T}_{4}$, el segundo grupo no recibió tratamiento $(\mathrm{n}=58)$ y las mujeres con anti-TPO negativos sirvieron como control $(\mathrm{n}=869)$. En ambos grupos, 
se midieron niveles de $\mathrm{TSH}_{\text {y }} \mathrm{T}_{4}$ séricos durante todo el período gestacional. El porcentaje de abortos en el grupo de pacientes con anticuerpos tiroideos positivos sin tratamiento con levotiroxina durante el primer trimestre de embarazo fue significativamente elevado, alcanzando 13\%. De acuerdo con ésto, el aborto fue la segunda complicación más frecuente después del parto pretérmino. También se observó cómo el tratamiento con levotiroxina redujo el riesgo de aborto cuando se suministró adecuadamente en las primeras etapas del embarazo en pacientes con hipotiroxemia. Igualmente, la tasa de partos pretérmino se redujo significativamente con la suplencia de la hormona tiroidea. ${ }^{26}$ Esto sugiere que el estado eutiroideo es muy importante en el inicio del embarazo y podría prevenir el aborto.

En un estudio prospectivo, Vaquero ${ }^{27}$ utilizó dos tratamientos diferentes para mujeres con anticuerpos anti-TPO y aborto recurrente con función tiroidea normal. El primer grupo de 11 mujeres fue tratado con Inmunoglobulina $\mathrm{G}$ intravenosa y el segundo grupo de 16 mujeres con extracto de hormona tiroidea. La tasa de abortos fue de $45 \%$ en el primer grupo de pacientes y de 19\% ( $p<0,05)$ en el grupo de mujeres tratadas con levotiroxina, lo que sugiere que el tratamiento con esta hormona en mujeres embarazadas con autoinmunidad tiroidea es benéfico. ${ }^{27}$ Por otra parte, en el estudio de Negro en mujeres eutiroideas con aborto recurrente y con anticuerpos anti-TPO se encontraron títulos de TSH ligeramente más altos que en las mujeres con aborto recurrente, sin estos anticuerpos, sugiriendo, en el primer grupo de pacientes, una menor reserva tiroidea o falla tiroidea leve. ${ }^{19,26}$ Dicha falla limitaría la respuesta a la mayor demanda de hormona tiroidea durante el embarazo. Puede ser, entonces, que la falla tiroidea leve explique, en parte, la asociación entre autoinmunidad tiroidea y aborto; y que en estas pacientes, la restitución hormonal temprana con levotiroxina pueda disminuir la recurrencia de aborto. Es por ésto, que a las mujeres embarazadas con enfermedad autoinmune tiroidea confirmada se les recomienda realizar un tamizaje prenatal temprano de TSH para detectar y prevenir, a tiempo, un estado de hipotiroidismo que conlleve a complicaciones para el feto y la madre. De acuerdo con los resultados obtenidos en el tamizaje, se recomienda iniciar restitución hormonal con levotiroxina logrando niveles de TSH menores a 2,5 $\mu \mathrm{U} / \mathrm{mL}$. Incluso, se debe realizar seguimiento de $\mathrm{T}_{4}$ libre durante el embarazo para tener un fundamento objetivo en el ajuste de las dosis de levotiroxina que se van a administrar. ${ }^{26}$

\section{RECOMENDACIONES}

De acuerdo con las recomendaciones realizadas en las guías para la práctica clínica, publicadas por la Sociedad de Endocrinología Clínica en lo relacionado con hipotiroidismo y embarazo, se sugiere: ${ }^{28}$

- Los hipotiroidismos fetal y materno tienen serios efectos adversos sobre el feto; por lo tanto, el hipotiroidismo materno debe evitarse, y su diagnóstico y manejo deben realizarse desde la primera visita.

- Si el hipotiroidismo se diagnostica desde antes del embarazo, se debe ajustar la dosis de tiroxina hasta alcanzar niveles pregestacionales de TSH no superiores a $2,5 \mu \mathrm{U} / \mathrm{mL}$.

- La dosis de $\mathrm{T}_{4}$ usualmente necesita aumentarse a la semana 4 - 6 y puede requerir un incremento de hasta $30-50 \%$.

- Si un hipotiroidismo clínico es diagnosticado durante el embarazo, la terapia de reemplazo debería lograr rápidamente niveles de TSH menores de 2,5 $\mu \mathrm{U} / \mathrm{mL}$ en el primer trimestre ó $3 \mu \mathrm{U} / \mathrm{mL}$ en el segundo o tercer trimestre. Las pruebas de función tiroidea deberían realizarse cada 30 días.

- Las mujeres eutiroideas con anticuerpos antitiroideos deberían vigilarse continuamente para detectar en forma temprana cualquier aumento en la TSH.

- Se recomienda terapia de reemplazo con $\mathrm{T}_{4}$ en pacientes embarazadas con hipotiroidismo subclínico (TSH elevada con niveles $\mathrm{T}_{4}$ libre normales). 
- Después del parto, la mayoría de mujeres necesitan disminuir la dosis de $\mathrm{T}_{4}$ que recibieron durante el embarazo.

- Aunque existe una asociación positiva entre la enfermedad tiroidea autoinmune y la pérdida del embarazo, no se recomienda una búsqueda universal para anticuerpos tiroideos, como tampoco es posible recomendar algún tratamiento en estos casos.

- Se recomienda una evaluación de la función tiroidea durante el embarazo en los siguientes casos:

1. Antecedente de enfermedad tiroidea.

2. Historia familiar de enfermedad tiroidea.

3. Mujeres con bocio.

4. Mujeres con anticuerpos antitiroideos.

5. Síntomas o signos sugestivos de disfunción tiroidea.

6. Mujeres con diabetes tipo 1 .

7. Mujeres con desórdenes autoinmunes.

8. Antecedente de irradiación de cabeza o cuello.

9. Historia de aborto o parto pretérmino.

\section{IMPLICACIONES PARA LA PRÁCTICA}

Aunque la asociación entre hipotiroidismo y aborto se ha mencionado en muchos textos, la relación causal no está completamente comprobada, como tampoco los fenómenos fisiopatológicos que explican esta asociación. Pese a que varios autores han reportado un aumento en el riesgo de aborto en las pacientes con hipotiroidismo clínico, hipotiroidismo subclínico y en pacientes eutiroideas con anticuerpos antitiroideos, fue en este último grupo donde se evidenció una fuerte asociación. Las razones expuestas para explicar estos hallazgos son: 1) una inadecuada adaptación de la función tiroidea a los mayores requerimientos hormonales necesarios en el embarazo, 2) la presencia de anticuerpos antitiroideos como marcador de una enfermedad autoinmune de base, y 3) el promedio de edad mayor en este grupo de pacientes (el lograr el embarazo tardíamente, como consecuencia de la asociación con infertilidad, implica para estas pacientes un mayor riesgo de aborto).
Además, se recomienda el diagnóstico y tratamiento tempranos del hipotiroidismo clínico y subclínico en las mujeres embarazadas, así como la búsqueda de anticuerpos antitiroideos en el grupo de abortadoras recurrentes.

Se requieren estudios adicionales con un mayor número de pacientes y un adecuado diseño para definir claramente la asociación entre aborto y enfermedad tiroidea, y así poder establecer terapias adecuadas.

\section{REFERENCIAS}

1. Glinoer D, Abalovich M. Unresolved questions in managing hypothyroidism during pregnancy. BMJ 2007;335:300-2

2. Krassas GE. Thyroid disease and female reproduction. Fertil Steril 2000;74:1063-70.

3. Pearce E, Farwell A, Braverman L. Thyroiditis. N Engl J Med 2003;348:2646-55.

4. Tamaky H, Amino N, Kimura M, Hidaka Y, Takeoka K, Miyai K. Low prevalence of thyrotropin receptor antibody in primary hypothyroidism in Japan. J Clin Endocrinol Metab 1990;71:1382-6.

5. Clavel S, Madec A, Bornet H, Deviller P, Stefanutti A, Orgiazzi J. Anti TSH-receptor antibodies in pregnant patients with autoimmune thyroid disorder. Br J Obstet Gynaecol 1990;97:1003-8.

6. Wilcox AJ, Weinberg CR, O'Conor JF, Baird DD, Schlatterrer JP, Canfield RE, et al. Incidence of early loss of pregnancy. N Engl J Med 1988;319:189-94.

7. Sagili H, Divers M. Modern management of miscarriage. The Obstetrician \& Gynaecologist 2007;9:102-8.

8. Francome C. Gynecologists and abortion in Northern Ireland. J Biosoc Sci 1994;26:389-94.

9. Vivas CA. Síndrome de ovario poliquístico, endometrio y riesgo de aborto. Rev Colomb Obstet Ginecol 2005;56:303-9.

10. Burrow GN, Fisher DA, Larsen PR. Maternal and fetal thyroid function. N Engl J Med 1994;331:1072-8.

11. Glinoer D. The regulation of thyroid function in pregnancy: pathways of endocrine adaptation from physiology to pathology. Endocr Rev 1997;18:404-33.

12. Smallridge RC, Ladenson PW. Hypothyroidism in pregnancy: consequences to neonatal health. J Clin Endocrinol Metab 2001;86:2349-53. 
13. Glinoer D, Gershengorn MC, Dubois A, Robbins J. Stimulation of thyroxine-binding globulin synthesis by isolated rhesus monkey hepatocytes after in vivo beta-estradiol administration. Endocrinology 1977;100:807-13.

14. Alexander EK, Marqusee E, Lawrence J, Jarolim P, Fischer GA, Larsen PR. Timing and magnitude of increases in levothyroxine requirements during pregnancy in women with hypothyroidism. N Engl J Med 2004;9:241-351.

15. Stagnaro-Green A, Roman SH, Cobin RH, el-Harazy E, Alvarez-Marfany M, Davies TF. Detection of atrisk pregnancy by means of highly sensitive assays for thyroid autoantibodies. JAMA 1990;264:1422-5.

16. Haddow JG Jr, Garbe PL, Miller DT. Maternal thyroid deficiency during pregnancy and subsequent neuropsychological development of the child. New Engl J Med 1999;341:2016-7.

17. Arteaga E. Función tiroidea y ciclo sexual femenino, fertilidad y embarazo. Bol. Esc. de Medicina, P. Universidad Católica de Chile 2000;29:125-8.

18. Lowe TW, Cunningham FG. Pregnancy and thyroid disease. Clin Obstet Gynecol 1991;34:72-81.

19. Poppe K, Glinoer D. Thyroid autoimmunity and hypothyroidism before and during pregnancy. Hum Reprod Update 2003;9:149-61.

20. LeBeau SO, Mandel SJ. Thyroid disorders during pregnancy. Endocrinol Metab Clin North Am 2006;35:117-36.

21. Abalovich M, Gutierrez S, Alcaraz G, Maccallini G, García A, Levalle O. Overt and subclinical hypothyroidism complicating pregnancy. Thyroid 2002;12:63-8.

22. Prummel MF, Wiersinga WM. Thyroid autoimmunity and miscarriage. Eur J Endocrinol 2004;150:751-5.

23. Wilson R, Ling H, McLean M, Mooney J, Kinnane D, McKillop J, et al. Thyroid antibody titer and avidity in patients with recurrent miscarriage. Fertil Steril 1999;71:558-61.

24. Matalon ST, Blank M, Levy Y, Carp H, Arad A, Burek L, et al. The Pathogenic role of anti-thyroglobulin antibody on pregnancy: evidence from an active immunization model in mice. Hum Reprod 2003;18:1094-9.

25. Muller AF, Verhoeff A, Mantel MJ, Bergohout A. Thyroid autoimmunity and abortion: a prospective study in women undergoing in vitro fertilization. Fertil Steril 1999;71:30-4.

26. Negro R, Formoso G, Mangieri T, Pezzarossa A, Dazzi D, Hassan H. Levothyroxine treatment in euthyroid pregnant women with autoimmune thyroid disease: effects on obstetrical complications. J Clin Endocrinol Metab 2006;91:2587-91.

27. Vaquero E, Lazzarin N, De Carolis C, Valensise H, Moretti C, Romanini C. Mild thyroid abnormalities and recurrent spontaneous abortion: diagnostic and therapeutic approach. Am J Reprod Immunol 2000;43:204-8.

28. Abalovich M, Amino N, Barbour LA, Cobin RH, De Groot LJ, Glinoer D, et al. Management of thyroid dysfunction during pregnancy and postpartum: an Endocrinol Society Clinical Practice Guideline. J Clin Endocrinol Metab 2007;92:S1-47. 